\title{
Isolated Spontaneous Renal Artery Dissection
}

Carrie Ng, MS III, Melissa Gitman, MD

\section{Case Report}

A previously healthy 37-year-old man presented to an outside hospital with an acute onset of sharp left lower quadrant pain that radiated into his groin while mowing the lawn. The pain was continuous, lasting until the patient presented to the emergency room of his local community hospital. He denied any trauma or sudden changes in position. The patient identified no aggravating or alleviating factors for the pain. The pain was associated with nausea and one episode of bilious, non-bloody emesis. He also noted urinary hesitancy but denied hematuria.

The patient's past medical history was significant for hyperlipidemia, controlled with gemfibrozil, and a 13-pack year smoking history. The patient presented to a local emergency department and underwent a CT scan of his abdomen that demonstrated an anterior left renal infarct due to a suspected thrombosis of anterior division of the left renal artery.
On transfer to the VA Hospital for further management, he was afebrile and normotensive. Physical examination was significant for an abdomen that was tender to palpation in the left lower quadrant and left costovertebral tenderness. Laboratory studies were notable for an elevated white blood cell count of 11500 per $\mathrm{mm}^{3}$ and a decreased platelet count of 97000 per cubic millimeter. His creatinine and blood urea nitrogen were within normal limits and were $1.0 \mathrm{mg} / \mathrm{dl}$ and $20 \mathrm{mg} / \mathrm{dl}$ respectively. During his hospital course, his blood pressure ranged between 128-140/81-84, his Cr remained stable, and he had one episode of low-grade fever of $100.1^{\circ} \mathrm{F}$. A magnetic resonance angiogram (MRA) was performed on day 2 of hospitalization (Figure 1).

\section{Discussion}

\section{Introduction}

The differential diagnosis for renal infarct includes embolic disease, thrombosis of the renal artery, renal artery dissection, and

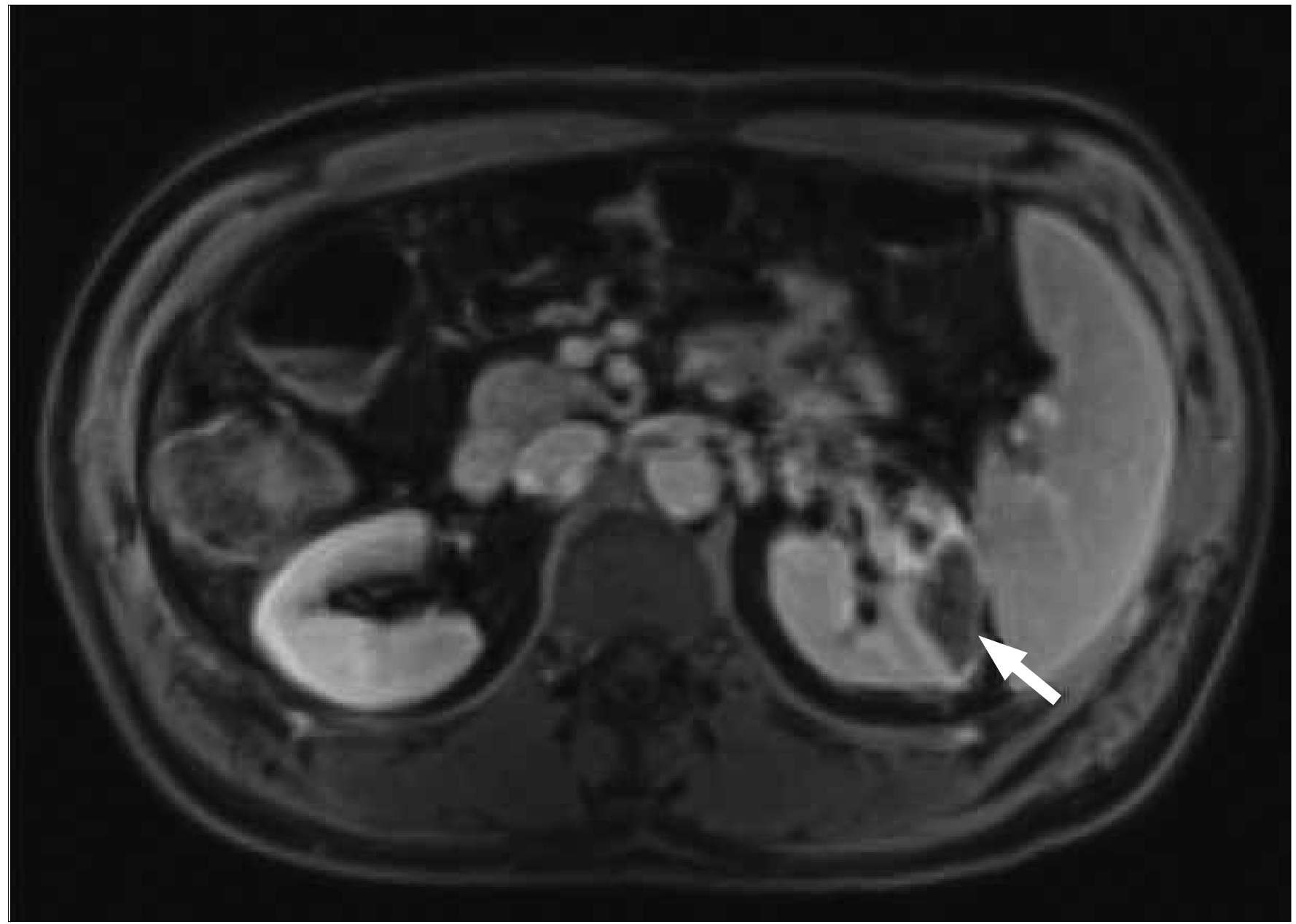

Figure 1. MRA demonstrating renal infarct. 
injury to the renal artery. Injury to the renal artery is responsibly for more than $75 \%$ of renal artery dissections and is generally due to extension of an aortic dissection, a trauma to the abdomen or an iatrogenic injury. The MRA the patient underwent on day two of his hospitalization confirmed the presence of a renal infarct but showed no focal aneurysm, dissection or stricture. Several diagnoses were entertained for possible etiologies of the renal infarct. The initial diagnosis of thrombosis was ruled out by the absence of clot on review of both the initial CT scan at the outside hospital as well as the MRA. The MRA failed to identify any abnormalities in the vasculature and serological studies did not demonstrate evidence of Polyarteritis Nodosa (PAN) thus this was felt to be unlikely to be the cause. An echocardiogram failed to demonstrate valvular vegetation and given the absence of other signs of embolic disease this diagnosis was ruled out. The diagnosis in this patient was finally reached by exclusion. Although the MRA did not demonstrate a dissection it was felt that, SRAD was the most plausible cause of his renal infarct given the patient's history and clinical presentation as evidenced by the discussion below.

Isolated SRAD is a rare event, with less than 200 reported cases in literature, and a quarter of those being autopsy findings. ${ }^{1}$ The actual incidence is higher than reported because many dissections are either silent or resolve spontaneously. ${ }^{1,2}$ As clinically apparent SRAD is uncommon, there is minimal data available concerning the optimal management strategy for this condition.

\section{Clinical Features}

The etiology of SRAD is often unknown. A few factors have been identified as risk factors for SRAD including atherosclerosis, fibromuscular dysplasia, connective tissue diseases and other conditions that might affect the integrity of the renal artery $^{1}$. The condition is more common among males, smokers and generally occurs between the ages of thirty to fifty. Most patients do not have other underlying co-morbid conditions. The most common clinical presentation is the acute onset of severe abdominal or flank pain. Another common finding is the presence of low-grade fever as seen in our patient. Much of literature on SRAD describes its clinical presentation as having severe hypertension and renal insufficiency. ${ }^{1,2}$ On the conrtrary, Ramamoorthy et al ${ }^{5}$ and Misrai et al ${ }^{4}$ suggest that most patients actually present like ours did with normal blood pressure and renal function with symptoms of kidney infarction.

\section{Work-up}

Diagnostic work-up should include checking a complete blood count for the presence of leukocytosis, basic metabolic panel to check renal function, liver function enzymes to rule out other causes of abdominal pain and a urinalysis for the presence of red blood cells. The diagnosis is not usually made until it is detected on radiologic imaging, because the history, physical, and laboratory findings results are usually non-specific. CT of the abdomen with contrast is generally the first imaging study ordered, usually undertaken for suspected nephrolithiasis.
While MRA may be useful in making the diagnosis, angiography is the goal standard and remains the modality of choice as it can potentially be diagnostic and interventional. ${ }^{1}$

\section{Treatment}

Once a SRAD is diagnosed, there are several options for treatment including medical, surgical, and interventional radiology depending on the renal function and lesion stability. Spontaneous resolution as seen in our case has been documented and observation or medical management of hypertension may be sufficient. ${ }^{1,2}$ Anticoagulation has also been suggested for medical management although it has not been demonstrated that this confers any long term benefit ${ }^{1}$. Surgical treatments such as vascular reconstruction, and endovascular procedures such as stenting or coil embolization, have also described in literature. ${ }^{1,3}$ Surgical interventions have been identified as beneficial in cases where the patient does not have significant renal ischemia but continues to suffer uncontrollable hypertension. Vascular reconstruction has been described as having good long-term results, but post-operatively many patients still require anti-hypertensives and the procedure has a high morbidity rate including acute thrombosis of the renal artery and late anastomotic restenosis. ${ }^{3,4}$ Revascularization with stenting is indicated when there is a severely stenotic vessel that provides blood flow to an ischemic part of the kidney. ${ }^{3}$ If patient has an isolated involvement of an accessory renal artery, then percutaneous embolization is another option. Finally, nephrectomy is considered when the kidney is already severely damaged from the infarction or if revascularization would be not be an option. ${ }^{1,5,6}$

\section{Conclusion}

The available literature suggest that SRAD is a relatively benign condition and surgical treatment are considered when patient has failed medical management. ${ }^{5}$ However, long-term follow-up of blood pressure and renal function are important since this condition is generally poorly understood. ${ }^{1}$ The patient in this case was managed conservatively with analgesics and anti-emetics and was discharged home without any sequelae of his renal infarct.

\section{References}

1. Kanofsky JA, Lepor H (2007) Spontaneous renal artery dissection. Reviews in Urology 9: 156-160

2. Mudrick D, Arepally A, Geschwind JF, Ronsivalle JA, Lund GB, Scheel P (2003) Spontaneous renal artery dissection: treatment with coil embolization. $J$ Vasc Interv Radiol 14: 497-500

3. Misrai V, Peyromaure M, Poiree S., Marteau V, Laurian C (2006) Spontaneous dissection of branch renal artery - is conservative management safe and effective? J Urol 176: 2125-2129

4. Pellerin O, Garcon P, Beyssen B, Raynaud A, Rossignol P, Jacquot C, Plouin PF, Sapoval M (2009) Spontaneous renal artery dissection: Long-term outcomes after endovascular stent placement. J Vasc Interv Radiol 20: 1024-1030

5. Ramamoorthy SL, Vasquez JC, Taft PM, McGinn RF, Hye RJ (2002) Nonoperative management of acute spontaneous renal artery dissection. Ann Vasc Surg 15:157-162

6. Lacombe M (2001) Isolated spontaneous dissection of the renal artery. J Vasc Surg 33: 385-391 